\title{
Model Based Cardiac Motion Tracking Using Velocity Encoded Magnetic Resonance Imaging*
}

\author{
Erik Bergvall ${ }^{1,2}$, Erik Hedström ${ }^{2}$, Håkan Arheden ${ }^{2}$, and Gunnar Sparr ${ }^{1}$ \\ ${ }^{1}$ Centre for Mathematical Sciences, Lund Institute of Technology, Lund, Sweden \\ ${ }^{2}$ Department of Clinical Physiology, Lund University Hospital, Lund, Sweden
}

\begin{abstract}
This paper deals with model based regularization of velocity encoded cardiac magnetic resonance images (MRI). We extend upon an existing spatiotemporal model of cardiac kinematics by considering data certainty and regularity of the model in order to improve its performance. The method was evaluated using a computer simulated phantom and using in vivo gridtag MRI as gold standard. We show, both quantitatively and qualitatively, that our modified model performs better than the original one.
\end{abstract}

\section{Introduction}

Cardiovascular disease is the main cause of death in the western world with left ventricular infarction as the predominant contributor to this phenomenon. The use of non-invasive and non-ionizing imaging techniques, such as echocardiography and MRI have aided in the diagnosis through the ability to directly visualize cardiac structure and function.

Quantitative assessment of regional myocardial function is a challenging but important task as subjective assessment of regional wall motion may suffer from poor inter-observer agreement [1. Several approaches have been developed for the quantitation of regional myocardial function using MRI. Saturation grid-tagging allows direct evaluation of myocardial deformation of the heart [2], 3], 4, [5] but is limited by relatively low spatial resolution and tag fading late in diastole [6, 7]. Further, specialized software for identification of tag lines is needed [8].

Another approach utilizes the velocity information present in phase contrast sequences providing velocity fields with high spatial and temporal resolution 9]. This velocity data may be used to directly calculate myocardial strain rate [10, 11, 12, 13] or can be integrated with respect to time, giving the motion of the myocardium. From this basal kinematic descriptor, a number of interesting mechanical properties, such as Lagrangian strain, may be derived, which may provide understanding of cardiac mechanics and diagnosis of disease.

The measured velocity field is subject to noise, imaging artifacts and degradation by sampling in time and space. The 'forward-backward' integration [14, [15]

\footnotetext{
* This work was supported by the Swedish Heart-Lung foundation and the Region of Scania.
} 
ensures a periodic motion but does not model any spatial coherence of the myocardium. Other approaches have used a deformable mesh guided by a Kalman filter, or combined with Fourier analysis to obtain both periodic motion and spatial smoothness [16], [17, [18, [19].

The purpose of this work is to develop a model based regularization strategy for velocity data measured using phase contrast MRI in order to be able to measure cardiac deformation and strain in a quantitative way. Zhu proposed the use of a cyclic spatiotemporal finite element model [19]. The elements in the model were constructed by piecewise linear functions and harmonics with varying frequency to ensure a periodic motion. The parameters in the model were determined by an iterative scheme consisting of updating the mesh configuration and projection of the sampled velocity onto the elements.

We extended upon this spatiotemporal model in a number of ways. Zhu et al used a moving mesh to define the spatial elements. These elements needed to be redefined and the stiffness matrix was reconstructed in every iteration. We instead described the deformation in Lagrangian coordinates which results in a fixed mesh and also a fixed stiffness matrix. Secondly, the use of piecewise continuous elements may be inappropriate as the velocity field in a solid in motion must be not only be continuous but also differentiable [20]. We therefore investigated other elements. Further, the projection of measured velocities onto a model does not necessarily imply smoothness if no constraints are imposed on the parameters. The measured velocity field may be locally corrupted by e.g. partial volume averaging. We therefore estimated the certainty of a given measurement and included this information when determining the model parameters.

\section{Methods}

\subsection{Image Acquisition}

Velocity data was acquired in both in-plane directions in long-axis slices in human subjects for a total of 20 acquisitions. A 1.5 T Gyroscan Intera Scanner (Philips Medical Systems) was used for the acquisitions. Acquisition time varied between 30-90 s. Spatial and temporal resolution was $1.5 \mathrm{~mm} \times 1.5 \mathrm{~mm} \times$ $10 \mathrm{~mm}$, with 32 time frames covering the whole cardiac cycle. Typical imaging parameters were repetition time $(\mathrm{TR})=24 \mathrm{~ms}$, echo time $(\mathrm{TE})=5.3 \mathrm{~ms}, v_{\text {enc }}$ $=0.20 \mathrm{~m} / \mathrm{s}$, flip angle $=20^{\circ}$, matrix size $=256 \times 192$ pixels and field of view $(\mathrm{FOV})=400 \mathrm{~mm} \times 300 \mathrm{~mm}$. Saturation bands $(30 \mathrm{~mm}$ thickness, $30 \mathrm{~mm}$ gap to image plane) superior and inferior to the imaging slice were applied to reduce signal from blood [21]. Retrospective gating was used when reconstructing the images. The boundary of the left ventricle was manually delineated in the first frame of each image sequence.

For validation purposes, saturation tagged images were acquired as a single breathhold sequence in the same imaging plane. Typical imaging parameters were: $\mathrm{TR}=3.8 \mathrm{~ms}, \mathrm{TE}=1.8 \mathrm{~ms}$, flip angle $=15^{\circ}$, saturation tag gap $=7 \mathrm{~mm}$, matrix size $=256 \times 192$ pixels, FOV $400 \mathrm{~mm} \times 300 \mathrm{~mm}$, resulting in a temporal resolution of $64 \mathrm{~ms}$. 


\subsection{Spatiotemporal Model}

Let $\mathbf{x}$ be the coordinate vector in $\mathbf{R}^{2}$, and time $t=[0, T]$. We assume that we have acquired an image sequence $I(\mathbf{x}, t): \mathbf{R}^{2} \times(0, T) \rightarrow[0,1]$ and a velocity field $\mathbf{v}(\mathbf{x}, t): \mathbf{R}^{2} \times(0, T) \rightarrow \mathbf{R}^{2}$. The measured image sequence and the velocity field will only be given at discrete points in spacetime but can be treated as functions defined on all of $\mathbf{R}^{2}$ by interpolation. We let $\Omega$ be the set of points, henceforth called particles, that occupies the left ventricle at time $t=0$ and let $\mathbf{x}$ denote the Lagrangian coordinate vector. The set $\Omega$ is given by manual delineation of the left ventricle in the images.

We are interested in the motion $\phi(\mathbf{x}, t): \Omega \rightarrow \mathbf{R}^{2}$ for all particles. It is given by the particle trace equation

$$
\frac{d \phi(\mathbf{x}, t)}{d t}=\mathbf{v}(\phi(\mathbf{x}, t), t) .
$$

The right hand side $\mathbf{v}(\mathbf{x}, t)$ is given by measurements by velocity encoded MRI, and is subject to noise and artifacts. Therefore we do not attempt to solve (11) for individual particles. Following an approach similar to the one of Zhu et al [19], we construct a spatiotemporal model of the deformation of the left ventricle. We construct a vector $\mathbf{G}(\mathbf{x})$ of length $N$ with spatial basis elements $g_{i}(\mathbf{x})$ to be defined shortly, and a vector $\mathbf{H}(t)$ of length $K$ with temporal basis elements of the form $h_{k}(t)=\exp 2 \pi j k / T$. The spatiotemporal model is constructed as

$$
\phi(\mathbf{x}, t)=\mathbf{x}+(\mathbf{G}(\mathbf{x}) \otimes \mathbf{H}(t)) \mathbf{c},
$$

where $\otimes$ denotes the Kronecker direct product and $\mathbf{c}$ is a coefficient matrix of size $N K \times 2$. By construction, $\phi(\mathbf{x}, t)$ will be periodic in $t$ which is useful when describing cardiac motion. The Lagrangian velocity is given by

$$
\frac{d \phi(\mathbf{x}, t)}{d t}=(\mathbf{G}(\mathbf{x}) \otimes(\mathbf{H}(t) \omega)) \mathbf{c},
$$

with $\omega$ a diagonal matrix with elements $2 \pi j k / T$. The goal is to adapt this model to data by choosing the coefficient matrix $\mathbf{c}$ in an appropriate way.

If (11) holds, then

$$
\mathcal{E}=\int_{0}^{T} \int_{\Omega}\left|\frac{d \phi(\mathbf{x}, t)}{d t}-\mathbf{v}(\phi(\mathbf{x}, t), t)\right|^{2} d \mathbf{x} d t=0,
$$

so the particle trace equation can be reformulated as minimization of $\mathcal{E}$. If we consider $\mathbf{v}(\phi(\mathbf{x}, t), t)$ as a fixed function, not depending on $\mathbf{c}$, we can differentiate $\mathcal{E}$ with respect to $\mathbf{c}$ and equate to zero to obtain a linear system

$$
\left(\int_{0}^{T} \int_{\Omega}(\mathbf{G} \otimes(\mathbf{H} \omega))^{\top}(G \otimes(\mathbf{H} \omega)) d \mathbf{x} d t\right) \mathbf{c}=\int_{0}^{T} \int_{\Omega}(\mathbf{G} \otimes(\mathbf{H} \omega))^{\top} \mathbf{v}(\phi(\mathbf{x}, t)) d \mathbf{x} d t .
$$


For ease of notation we let the left hand side stiffness matrix be denoted by $\mathbf{K}$ and the right hand side by $\mathbf{b}$ so that (3) reads

$$
\mathbf{K c}=\mathbf{b} .
$$

Now, minimization of $\mathcal{E}$ can be accomplished by iterative sampling of $\mathbf{v}(\mathbf{x}, t)$ at $\mathbf{x}=\phi(\mathbf{x}, t)$, and updating the model parameters by solving (4).

\subsection{Choice of Spatial Elements}

There is a large selection of basis elements to choose from. A common choice of elements are the piecewise linear interpolation functions, as used by Zhu et al 19. This choice may be inappropriate as the resulting function is not differentiable which violates the property that a deformation of a body is required to be in $C^{2}$ with respect to both space and time [20].

Our use of the elements is quite different from the use in e.g. the finite element method (FEM) where the purpose is to approximate a function as well as possible. In FEM applications it is common to refine a solution by adding and/or reshaping elements. In this application, a refinement would potentially lead to a less regular solution as the increased number of degrees of freedom will make it easier for the model to adapt to noise and artifacts. A similar reasoning applies to elements with a small support.

Thin plate splines (TPS) are a class of widely used non-rigid mappings, and are often used in computer vision tasks such as image registration or warping. The TPS is given by 22,

$$
g(\mathbf{x})=|\mathbf{x}|^{2} \ln \mathbf{x},
$$

which is a $C^{2}$ function outside the origin. We construct the vector $\mathbf{G}$ in (2) as $\mathbf{G}(\mathbf{x})_{i}=g\left(\mathbf{x}-\mathbf{x}_{i}\right)$, where the points $\mathbf{x}_{i}, i=1, \ldots, N$ are placed on the boundary of $\Omega$. By this we obtain a model that generates a $C^{2}$ mapping that can be locally controlled by the coefficient matrix $\mathbf{c}$.

\subsection{Extension of the Spatiotemporal Model}

The right hand side in (4) is constructed using measurements. It would therefore be a good idea to estimate a certainty of $\mathbf{v}(\phi(\mathbf{x}, t))$. We formally construct it as $w(\mathbf{x}, t): \mathbf{R}^{2} \rightarrow[0,1]$, and redefine $\mathcal{E}$ as a weighted functional

$$
\mathcal{E}=\int_{0}^{T} \int_{\Omega}\left(\frac{d \phi(\mathbf{x}, t)}{d t}-\mathbf{v}(\phi(\mathbf{x}, t), t)\right)^{2} w(\phi(\mathbf{x}, t), t) d \mathbf{x} d t=0 .
$$

The explicit construction of $w(\mathbf{x}, t)$ will be discussed below. This will also lead to a linear system, which will be similar to (3). We construct the weight function by considering the local variation of the vector field around a given point $\phi\left(\mathbf{x}_{k}, t\right)$ and let

$$
w\left(\phi\left(\mathbf{x}_{k}, t\right)=\exp \left(-\int_{B_{r} \cap \Omega}\left(\mathbf{v}(\phi(\mathbf{x}, t))-\mathbf{v}\left(\phi\left(\mathbf{x}_{k}, t\right)\right)\right)^{2} d \mathbf{x} / \sigma^{2}\right),\right.
$$


where $B_{r}\left(\mathbf{x}_{k}\right)$ is a neighborhood of $\mathbf{x}_{k}$ and $\sigma$ is a tunable parameter. Here we note that the variation is defined for the measured velocities sampled along the estimated particle traces. As these velocities are supposed to be sampled within the myocardium, we do not expect them to have a large variation within a given neighborhood.

Even if we use a motion model, it does not necessarily generate what we, in some sense, mean by a regular deformation. If the coefficient matrix can be chosen arbitrarily it would be easy to generate deformations that are unreasonable from both a physiological and a physical point of view. To avoid such unwanted behavior we propose an additional regularity term in the energy functional. We construct it as

$$
\mathcal{E}_{\text {regularity }}=\int_{0}^{T} \int_{\Omega}|\Delta \phi(\mathbf{x}, y)|^{2} d \mathbf{x} d t,
$$

where $\Delta$ is the spatial Laplace operator. This regularity term only affects the spatial part of the deformation. The temporal part can be regularized by e.g. using only few harmonics in the model. Further, the regularizing term does not penalize affine transformations and as a consequence does not penalize rigid transforms. This is an important property, as otherwise the regularity term would depend on our choice of coordinate system. The addition of this term will transform (4) to

$$
\mathbf{K} \mathbf{c}+\lambda \mathbf{L} \mathbf{c}=\mathbf{b},
$$

where $\mathbf{L}_{i j}=\int_{\Omega} \Delta g_{i}(\mathbf{x}) \Delta g_{j}(\mathbf{x}) d \mathbf{x}$ and $\lambda>0$ is a tunable parameter that determines the influence of the regularity term.

\subsection{Experimental Validation}

In a first experiment we constructed a computer generated phantom based on a kinematic model of the left ventricle described by Arts et al [23. This model was used to generate Lagrangian motion as a gold standard and also Eulerian velocities which was used as input data. The pixel dimensions and temporal resolution of the model-generated data was chosen to be similar to measured velocity data. The Eulerian velocities were corrupted by Gaussian noise with standard deviation ranging from 0 to 5 pixels/frame which should be compared to the peak velocities of about 2 pixels/frame. The velocities outside the deforming body were generated as zero mean Gaussian noise with a standard deviation of 5 pixels/frame.

In a second experiment we used the acquired gridtag images as gold standard. Gridtag sequences were analyzed manually and tagline intersections were tracked manually through all time frames and for all image series acquired.

We investigated several versions of the spatiotemporal model. As a reference we used the case with piecewise linear elements with no data certainty estimate or regularization parameter. This will be close to the method proposed by Zhu et al 19. In the other experiments we used TPS elements and combinations of weighted fit and regularity term. The parameters used were $\sigma=0.1$ pixels/frame, 
$\lambda=100$ and the size of the neighborhood $B_{r}$ was 3 pixels. We typically use $N=25$ spatial basis elements and 10 iterations in the algorithm.

The root mean square (RMS) was used to measure the error between the particle traces estimated by our proposed method and the Lagrangian motion given by the kinematic model in the first experiment and the manually constructed particle traces in the second experiment.

\section{Results}

Figure 1 shows the result of motion tracking using simulated data. The linear element model and the TPS model without adjustments perform in a similar way and the best model is the TPS model with weighted fit and a regularity term.A qualitative comparison of the case with piecewise linear elements and the case with TPS elements with regularity term are shown in Figure 2. The TPS based model generates a smoother deformation than the other model. It is possible to see drastic changes in the deformation in the linear elements case which are due to the tessellation of the domain. In absolute terms the methods perform in a

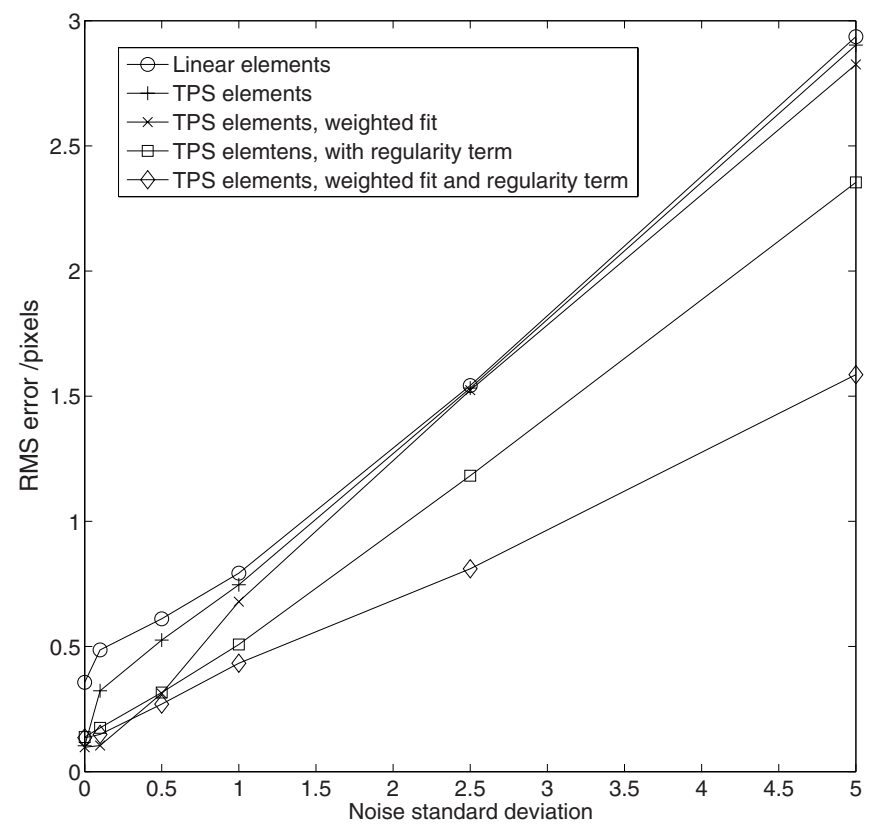

Fig. 1. The figure shows the motion tracking error for the simulated phantom for different levels of Gaussian noise. It can be seen that linear element model and the TPS model without adjustments are the worst performers. For higher levels of noise the weighting term flattens out and treats all noisy measurements in the same way which explains the appearance of the curve. Thus, it becomes essential that a regularity term is added when the noise level is high. 

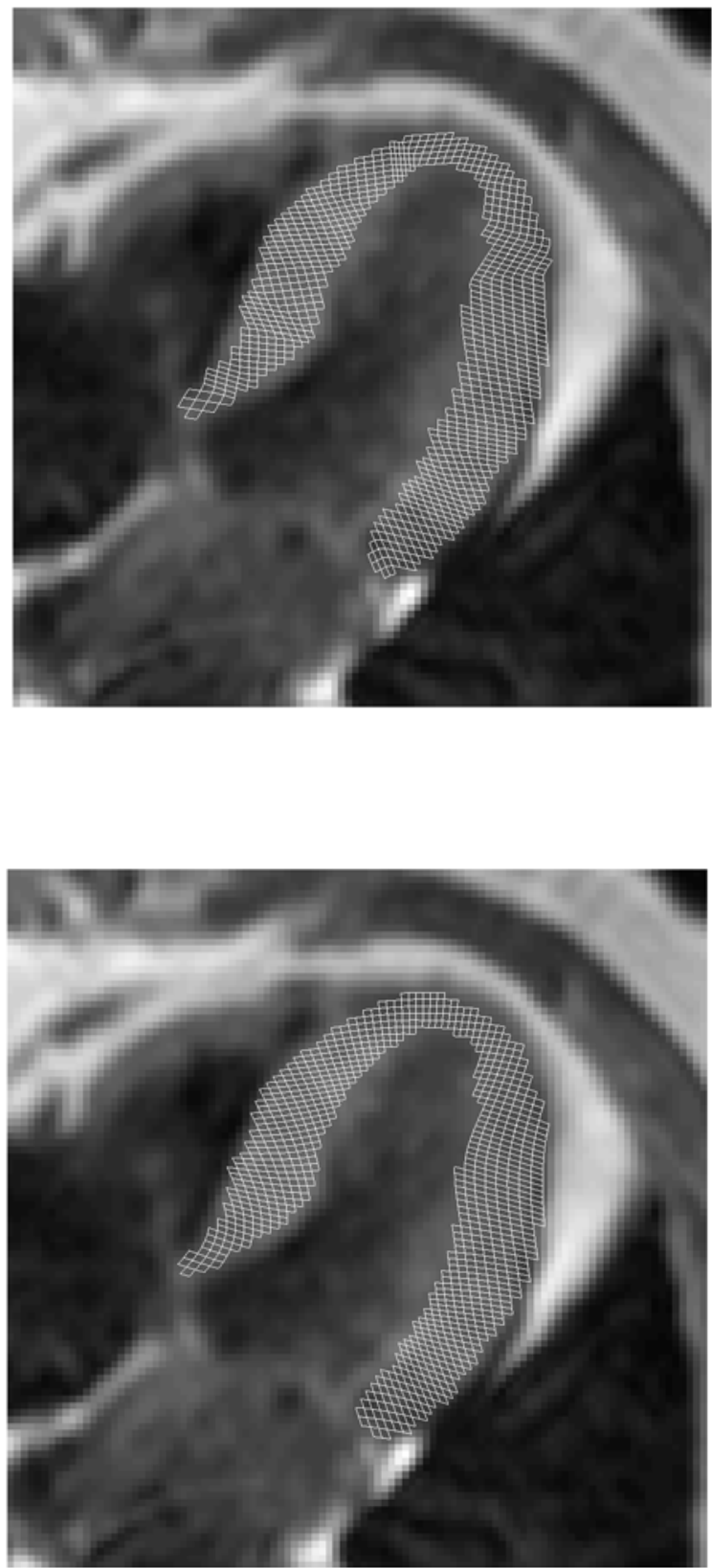

Fig. 2. A qualitative comparison of a spatiotemporal model with piecewise linear elements (top) and a model with TPS elements and additional regularity term (bottom). The deformation is shown at end systole (peak of contraction). The TPS based model generated a smoother deformation than the other model. It is possible to see drastic changes in the deformation in the top figure which are due to the tessellation of the domain. 


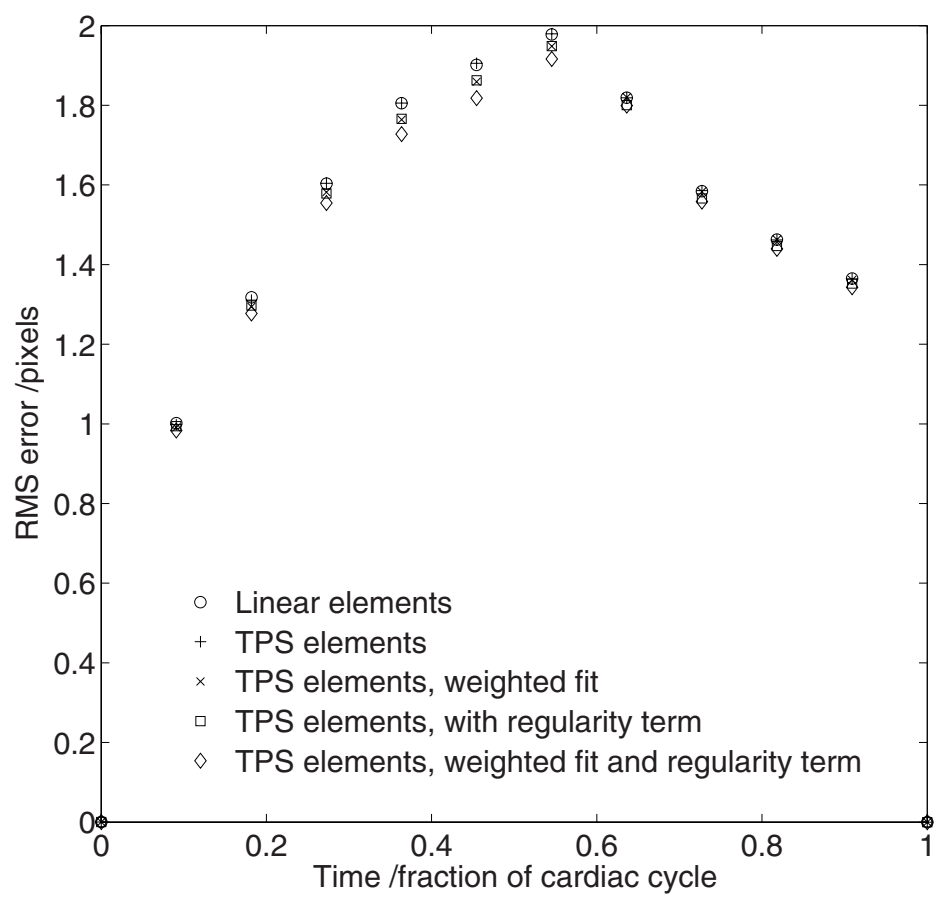

Fig. 3. A quantitative comparison of the different spatiotemporal models. The figure gives the mean RMS error for the 20 cases compared to grid tag MRI. The absolute difference between the different variants is small which is due to the sparsity of the displacement obtained from the grid tag images. The error is higher during the second half of the cardiac cycle which is due to grid tag fading. Note that the model using linear elements has a higher error than all the other methods. The model with the smallest error is the one with both a data certainty term and a regularity term.

similar way, the difference between particle traces generated by the two models will often be on the order of a pixel or less. This difference is, however, enough to generate quite different deformations, if evaluated in a qualitative way.

Figure 3 shows the RMS error for the different models. The best performer was the model with both data certainty and regularity terms. Again, the absolute differences between the models were quite small.

\section{Discussion}

In this paper we have extended a spatiotemporal model of cardiac deformation proposed by Zhu et al [19] in several ways. First, we made the observation that piecewise linear elements are inappropriate as they are not differentiable. Therefore we used the TPS as a spatial element. Second, we estimated a data certainty term so that the model does not adapt to noisy measurements. Third, we also added a regularity term so that smooth deformations are encouraged. 
We also defined the model in Lagrangian coordinates which is beneficial from a computational point of view.

Based on the qualitative and quantitative results presented we conclude that all variants of our proposed model performs better than, or equally well, as the model of Zhu et al. The adjustments made to the model have proven to be beneficial as showed using a computer generated motion phantom where the RMS error can be up to $50 \%$ lower for our proposed model. There are however, only small quantitative differences between the model variants when evaluated in vivo. Part of this can be explained by our validation procedure where we used gridtag MRI as a gold standard. Grid tag MRI will only provide a sparse displacement field which means that the regularity of the calculated deformation will not be reflected in the error. The grid tag images were manually analyzed which is an additional source of error, in particular during diastole where tag fading complicates the analysis. This effect can be seen in Figure 3 where the error is higher during the second half of the cardiac cycle. Figure 2 showed that the absolute difference between the deformations generated by the model will be small, which is reflected in the error shown in Figure 3 . The use of in vitro measurements would be helpful in order to determine the best model variant and is therefore the focus of future work.

\section{References}

1. Picano, E., Lattanzi, F., Orlandini, A., Marini, C., L'Abbate, L.: Stress echocardiography and the human factor: the importance of being expert. Journal of the American College of Cardiology 83, 1262-1265 (1991)

2. McVeigh, E., Ozturk, C.: Imaging myocardial strain. IEEE Signal Processing Magazine 18(6), 44-56 (2001)

3. O’Dell, W.G., Moore, C.C., Hunter, W.C., Zerhouni, E.A., McVeigh, E.R.: Threedimensional myocardial deformations: Calculation with displacement field fitting to tagged MR images. Radiology 195(3), 829-835 (1995)

4. Axel, L., Dougherty, L.: MR imaging of motion with spatial modulation of magnetization. Radiology 171, 841-845 (1989)

5. Zerhouni, E.A., Parish, D.M., Rogers, W.J., Yang, A., Shapiro, E.P.: Human-hearttagging with $\mathrm{mr}$ imaging - a method for noninvasive assessment of myocardial motion. Radiology 169(1), 59-63 (1988)

6. McVeigh, E.: MRI of myocardial function: Motion tracking techniques. Magnetic Resonance Imaging 14, 137-150 (1996)

7. Masood, S., Yang, G., Pennell, D.J., Firmin, D.N.: Investigating intrinsic myocardial mechanics: The role of MR tagging, velocity phase mapping and diffusion imaging. Journal of Magnetic Resonance Imaging 12(6), 873-883 (2000)

8. Osman, N.F., McVeigh, E.R., Prince, J.L.: Imaging heart motion using harmonic phase MRI. IEEE Transactions on Medical Imaging 19(3), 186-202 (2000)

9. Pelc, N.J., Herfkens, R.J., Shimakawa, A., Enzmann, D.R.: Phase contrast cine magnetic resonance imaging. Magnetic Resonance Quarterly 7(4), 229-254 (1991)

10. van Wedeen, J.: Magnetic resonance imaging of myocardial kinematics. techniques to detect, localize and quantify strain rates of the active human myocardium. Magnetic Resonance in Medicine 27(1), 52-67 (1992) 
11. Robson, M.D., Constable, R.T.: Three-dimensional strain-rate imaging. Magnetic Resonance in Medicine 36(4), 537-546 (1996)

12. Arai, A.E., Gaither III, C.C., Epstein, F.H., Balaban, R.S., Wolff, S.D.: Myocardial velocity gradient imaging by phase contrast MRI with application to regional function in myocardial ischemia. Magnetic Resonance in Medicine 42(1), 98-109 (1999)

13. Selskog, P., Heiberg, E., Ebbers, T., Wigström, L., Karlsson, M.: Kinematics of the heart: Strain-rate imaging from time-resolved three-dimensional phase contrast MRI. IEEE Transactions on Medical Imaging 21(9), 1105-1109 (2002)

14. Constable, R.T., Rath, K.M., Sinusas, A.J., Gore, J.C.: Development and evaluation of tracking algorithms for cardiac wall motion analysis using phase velocity MR imaging. Magnetic Resonance in Medicine 32(1), 33-42 (1994)

15. Pelc, N.J., Drangova, M., Pelc, L.R., Zhu, Y., Noll, D.C., Bowman, B.S., Herfkens, R.J.: Tracking of cyclic motion using phase contrast cine mri velocity data. Journal of Magnetic Resonance Imaging 5(3), 339-345 (1995)

16. Meyer, F.G., Constable, T., Sinusas, A.J., Duncan, J.S.: Tracking myocardial deformation using phase contrast MR velocity fields: A stochastic approach. IEEE Transactions on Medical Imaging 15(4), 453-465 (1996)

17. Zhu, Y., Drangova, M., Pelc, N.J.: Fourier tracking of myocardial motion using cine-PC data. Magnetic Resonance in Medicine 35(4), 471-480 (1996)

18. Zhu, Y., Drangova, M., Pelc, N.J.: Estimation of deformation gradient and strain from cine-PC velocity data. IEEE Transactions on Medical Imaging 16(6), 840-851 (1997)

19. Zhu, Y., Pelc, N.J.: A spatiotemporal model of cyclic kinematics and its application to analyzing nonrigid motion with MR velocity images. IEEE Transactions on Medical Imaging 18(7), 557-569 (1999)

20. Chadwick, P.: Continuum Mechanics. Dover Publications, Mineola (1999)

21. Drangova, M., Zhu, Y., Pelc, N.J.: Effects of artifacts due to flowing blood reproducibility of phase-contrast measurements of myocardial motion. Journal of Magnetic Resonance Imaging 7(4), 664-668 (1997)

22. Bookstein, F.L.: Principal warps: thin-plate splines and the decomposition of deformations. IEEE Transactions on Pattern Analysis and Machine Intelligence 11(6), 567-585 (1989)

23. Arts, T., Hunter, C., Douglas, A., Muijtens, M.M., Reneman, R.: Description of the deformation of the left ventricle by a kinematic model. Journal of Biomechanics 25(10), 1119-1127 (1992) 